\title{
System Design and Control Research for New Quadrotor Searching and Saving Aircraft
}

\author{
Wang Qi ,Yuan Mingxin,Zhang Peng \\ School of Mechatronics and Automobile Engineering \\ Jiangsu University of Science and Technology \\ Zhangjiagang, China,215600 \\ wqi003@126.com
}

\author{
Cheng Fei ,Cao Yuqiang \\ School of Mechatronics and Automobile Engineering \\ Jiangsu University of Science and Technology \\ Zhangjiagang, China,215600
}

\begin{abstract}
In order to realize on-side rescue after destructive natural devastation or biological and chemical contamination, a new quadrotor aircraft is designed, which can fly into some dangerous areas to detect and search. This system is consist of controller, gesture detection, quadrotor motor and drive, wireless controller, wireless video capturing and date transmission module, etc. Wireless correspondence based on blue tooth implements the remote terminal control in the monitoring center. At the same time, the wireless camera equipped in the aircraft helps to achieve the remote site monitoring, aerial photography and mapping. The experiment result verifies the feasibility of designed system.
\end{abstract}

Keywords- Quadrotor, Searching and Saving, Aircraft, Wireless Correspondence, Wireless Video

\section{INTRODUCTION}

China has vast and complicated natural conditions, and it is one of the countries that frequently suffer from the ravages of natural disasters. In January, 2008, China suffered a huge snow disaster once ever in 50 years. Not so long after the snow disaster, the city Wenchuan in Sichuan province suffered an 8 magnitude earthquake on May 12th 2008.Two years later, on April 14th.2010, a 7.2-magnitude earthquake hit the city Yushu in Qinghai province again. Ice after the snow and storm made the line checking and repairing much more difficult. While the Landslides and debris flows not only brought difficulty for rescue, but also increased the likelihood that people got injured in the rescue. Apart from natural calamities, biological and chemical contamination was getting much worse, poisonous gas leaks happened when workers were taking apart waste cylinders at a recycle station in Qiqihaer in 2008, and in Wuxi 2010, poisonous gas leaks in one chemical company.

Although we can't prevent natural disasters and the emergence of biological and chemical pollution is inevitable as well. It is feasible to decrease the damage brought by the disasters and pollutions. Recent years, how to detect and rescue in unreachable areas is becoming hotter, and unmanned aircraft is a research focus due to its smaller size, lighter body, and easy-approach to dangerous areas, but it is also intricacy because it is really difficult to control and manoeuvre.

Some developed countries in Europe and South America lead the research of micro aircraft [1]. For instance, the "black widow" developed by American Airlines
Environmental Company, the "micro bat" developed by American Airlines Environmental Company and California Institute of Technology, and "tutor" developed by SRI International of the United States and University of Toronto. In China, researches are mainly done by well known institutes, such as Shanghai JiaoTong University, National Defense University, JiLing University and so on. Robotics Laboratory of National Defense University in 2004 launched a micro quadrotor helicopter technology research.

To solve the on-site detection and rescue after major natural disasters and biological and chemical pollution, a new searching and saving quadrotor aircraft system is designed independently in this paper. The system has not only the basic flight capabilities, but a collection of wireless remote control, video capture and wireless transmission functions. Figure 1 shows the engineering applications of this paper. From the figure, it is shown that the aircraft can be remotely controlled to fly to the disaster scene in the monitoring center via Bluetooth, and then the on-site video will be wirelessly transmitted to the monitoring center to guide the on-site detection and rescue.

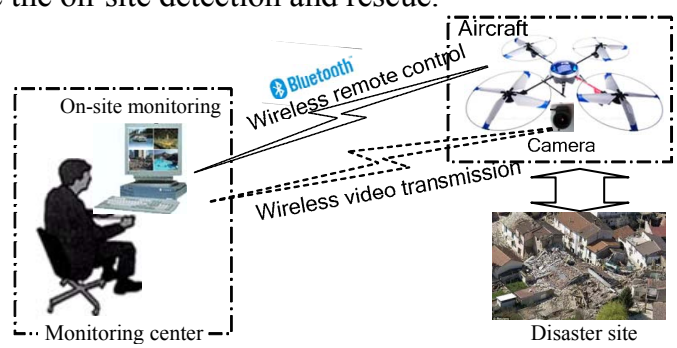

Figure 1. Application scheme in this paper

\section{The ELEMENTS Of Four-AXIS SEARCH AND RESCUE AIRCRAFT'S SYSTEMS}

In order to achieve post-disaster search and rescue scene, a new quator aircraft is designed in this paper. The system components shown in Figure 2 is mainly consist of the controller, gesture detection module, wireless remote control module, wireless video transmission module, rotor motor and drive modules and other components. 


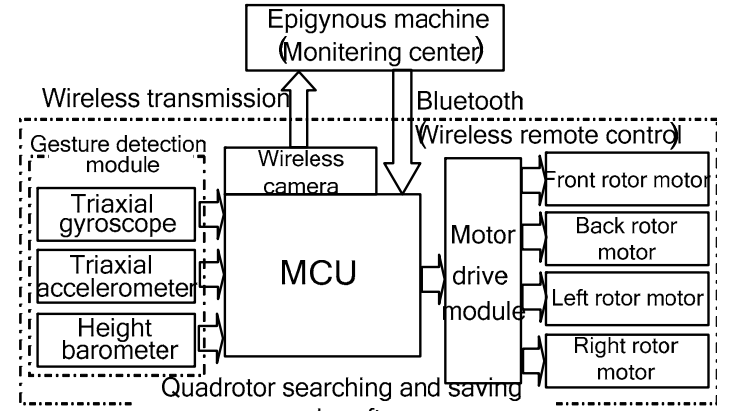

Figure 2. System components of quadrotor aircraft

\section{A. Controller}

Considering the aircraft's power problems, the system controller uses the MSP430 F2274. The MCU system is equipped with the required $\mathrm{I} / \mathrm{O}$ ports, timers, serial communication interfaces and so on.

\section{B. Gesture detection module}

In order to attain quadrotor aircraft's gesture control, the gesture detection is prerequisite and critical to the control system. The detection module of this aircraft system mainly consists of the triaxial gyroscoper, triaxial accelerometer, and height barometer.

Triaxial gyroscoper

In order to get aircraft's angular velocity respectively in three directions, namely pitch rate, roll angle rate, and yaw angular rate, the muRata (Murata) produced ENC-03R type gyro angular velocity is used in this system, and the designed peripheral circuits of the Triaxial gyroscoper is shown in Figure 3 (a).

Triaxial accelerometer

Since triaxial gyroscoper has the temperature drift phenomenon, with the temperature arising, the measurements will be away from their actual value. It requires the data measured by its correction and compensation. This system uses a freescale produced MMA7260-type acceleration sensor [4] to correct the value. And the designed peripheral circuits of the MMA7260 accelerometer are shown in Figure 3 (b).

Height barometer

In order to control the aircraft's altitude, the system uses the atmospheric pressure sensor and the MPX4115 sensor of freescale when initially designed, and the designed peripheral circuits of the height barometer is shown in Figure 3 (c).

\section{Wireless remote control module}

In order to help the monitoring center control the aircraft in a long distance more effectively, the Bluetooth communication module is used in the system design. The Bluetooth transmission module is connected to the aircraft's Msp430 F2274 chip to realize the communication between the host PC and the aircraft. PC sends control commands to the flight control board's Bluetooth receiver module via Bluetooth transmitter, Bluetooth receiver module transmits the command to the 2274 chip through the UART communication protocol, and then the $2274 \mathrm{MCU}$ transmits PPM wave to the gesture control module to adjust the flying aircraft, The connection between the Bluetooth receiver module and MSP430 F2274 microcontroller is described in Figure 3 (d).

\section{Wireless video transmission module}

In order to make monitoring center in real time about the aircraft's operation process, especially when it reaches the disaster scenes, the system using COMS 208 wireless camera. the COMS chip comes with the compression algorithm helps to get picture data makes wireless transmission of data easy, and the data transfer uses FM modulation, frequency in $1.2 \mathrm{GHz}$, bandwidth can be $18 \mathrm{M}$, after demodulation, the receiver will extract the image, and extracting the resulting image format for PAL system. And add the image to the USB communication protocol serially through image acquisition card, PC read COM port data and displays it on the $\mathrm{PC}$ interface.

\section{E. Rotor motor and driver modules}

The system selects BL2212/13 brushless DC motor due to the requirement of low cost and light weight. The operating voltage of A2915 brushless DC motor is 7.2 16.8VDC, current $1 \sim 35 \mathrm{~A}$, by changing the motor armature voltage and power cycle connected time ratio (that is, the duty cycle) to change the size of the average voltage to control motor speed. Motor driving module is mainly generated by a PWM wave chip, adjustable PWM wave control of three-phase full-bridge brushless DC motor drive circuit, which drives the motor rotate, The three-phase fullbridge brushless DC motor drive circuit is shown as Figure 3 (e).Figure 4 shows a finally designed quadrotor searching and saving aircraft in this paper. From the figure, you can see that the system uses four DC motors driving four rotors rotate to provide power to the aircraft. Aircraft is equipped with a Bluetooth receiving module and camera, so the wireless remote control and wireless video transmission can be realized.

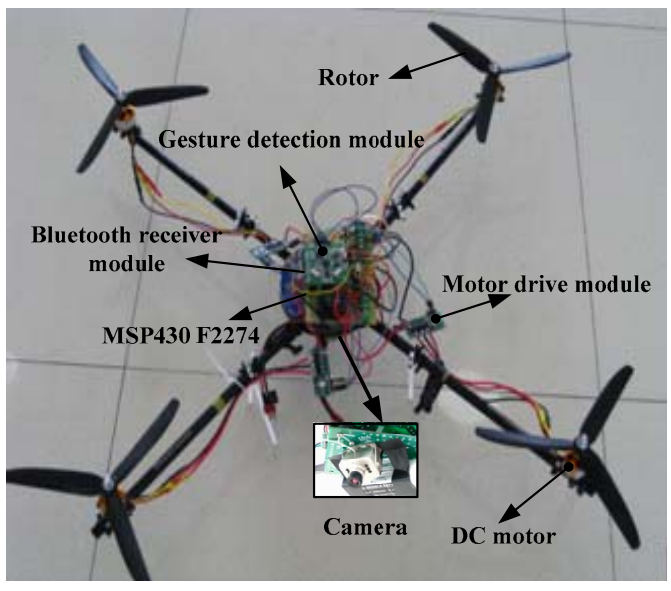

Figure 3. Designed aircraft in this paper 


\section{The Control Strategy Adjustment Of The QuADrotor SEARCHING AND SAVING AIRCRAFT}

In order to achieve effective control for the quadrotor aircraft in the air, a more sophisticated digital incremental PID control technology is used in this paper. The control variables includes the main pitching $(\mathrm{PITCH},(\mathrm{p})$ notes), roll angle (ROLL, (r) Note), and the deviation of the $\mathrm{x}, \mathrm{y}, \mathrm{z}$ direction. Full control of policy abbreviations are as follows:

$$
\begin{gathered}
U_{k}=U_{k-1}+\sum \Delta U_{k}=U_{k-1}+\Delta U_{k}(P)+\Delta U_{k}(R) \\
\quad+\Delta U_{k}(x)+\Delta U_{k}(y)+\Delta U_{k}(z) \\
\Delta U(P)=K_{p}(P) \cdot \Delta e_{k}(P)+K_{i}(P) \cdot e_{k}(P)+K_{d}(P) \cdot \Delta^{2} e_{k}(P) \\
\Delta U(R)=K_{p}(R) \cdot \Delta e_{k}(R)+K_{i}(R) \cdot e_{k}(R)+K_{d}(R) \cdot \Delta^{2} e_{k}(R) \\
\Delta U(x)=K_{p}(x) \cdot \Delta e_{k}(x)+K_{i}(x) \cdot e_{k}(x)+K_{d}(x) \cdot \Delta^{2} e_{k}(x) \\
\Delta U(y)=K_{p}(y) \cdot \Delta e_{k}(y)+K_{i}(y) \cdot e_{k}(y)+K_{d}(y) \cdot \Delta^{2} e_{k}(y) \\
\Delta U(z)=K_{p}(z) \cdot \Delta e_{k}(z)+K_{i}(z) \cdot e_{k}(z)+K_{d}(z) \cdot \Delta^{2} e_{k}(z) \\
\Delta e_{k}=e_{k}-e_{k-1} \\
\Delta^{2} e_{k}=e_{k}-2 e_{k-1}+e_{k-2}=\Delta e_{k}-\Delta e_{k-1}
\end{gathered}
$$

Where, $\mathrm{k}$ is the sample serial number; UK is the computer output values for $\mathrm{k}$ sampling time; $K_{P}(s=P, R, x, y, z)$ is the pitching, rolling point, $\mathrm{x}, \mathrm{y}$, and z scaling factors respectively; $K_{i}(s=P, R, x, y, z)$ is the integral coefficients of Pitch angle, roll angle and the $\mathrm{x}, \mathrm{y}$, $\mathrm{z}$ axis; $K_{d}(s=P, R, x, y, z)$ is the differential coefficient of pitching, rolling point and the axis of $\mathrm{x}, \mathrm{y}, \mathrm{z}$ Respectively. $e_{k}(s=P, R, x, y, z)$ is the gap between measured value and the actual expectations of Pitch angle.

Figure 5 shows how to control the gesture using PID controller based on Kalman filtering realize its function.

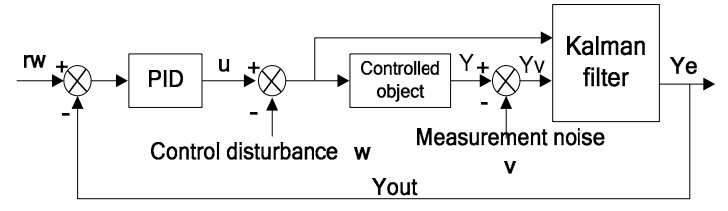

Figure 4. PID regulator based on Kalman filter

\section{SYSTEM EXPERIMENT}

In order to verify the feasibility of the designed quadrotor searching and saving aircraft, we carried out some indoor and outdoor flight experiments on the basis of completion of the related hardware and structure of aircraft.

\section{A. Indoor experiment}

This experiment is mainly performed in the laboratory, through Bluetooth communications module, using monitor host to send control commands to achieve flight test aircraft. Figure 6 gives the laboratory experiment process. Figure 6 (a) presents the initial time of aircraft and aircraft take off under the control of order that it increases the duty to improve the speed of four-axis motors, figures 6 (b)-(f) diagram show the

aircraft's lifting process, and figures 6 (g)-(i) show the aircraft can stay stable in the air. The whole indoor experiment verifies the feasibility of designed aircraft systems. However, considering the indoor environment is ideal. For example, the interference is relatively slight, especially where is no wind, which is essential for the search and rescue aircraft, so we still have to make more experiments to verify the outdoor aircraft.

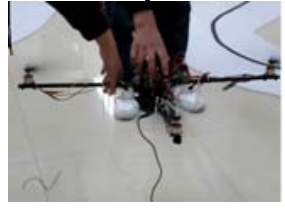

(a)

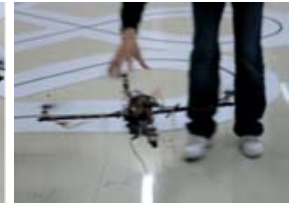

(b)

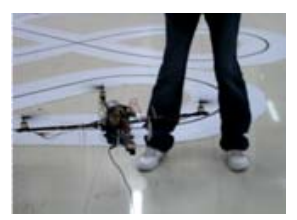

(c)

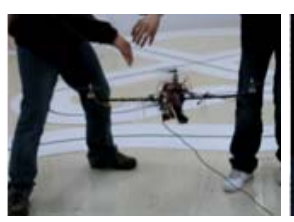

(d)

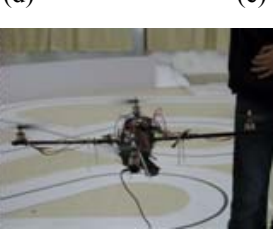

(f)

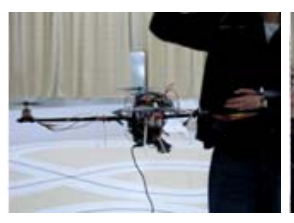

(g)

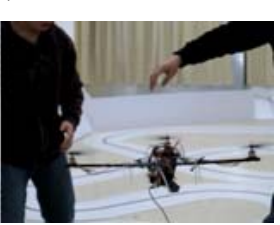

(e)

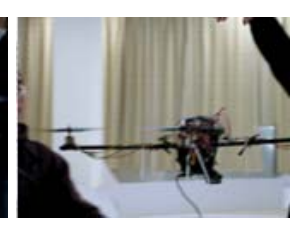

(h)

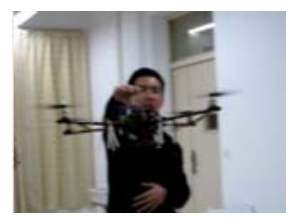

(i)

Figure 5. Experiment results in indoor

\section{B. Outdoor experiment}

The outside experiment is mainly performed on the playground, and the remote control is also used on the vehicle by the monitor host. The whole course is shown in 
the figure 7. Figure 7(a) is initial movement, figures 7 (b) (e) are the parts of launch, figures 7(f)--(h) show that the vehicle successfully keeps stable when floating in the air .figure (i) shows that the vehicle is soring in air stably. The left corner partial views of the (b) - (i) figures are the pictures of monitoring transmissed from the WIFI passage by the vehicle camera. In this paper, we can find that the rescue vehicle system designed in this book is feasible.

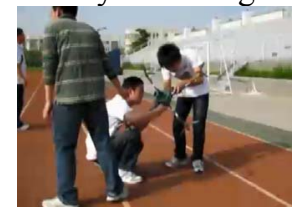

(a)

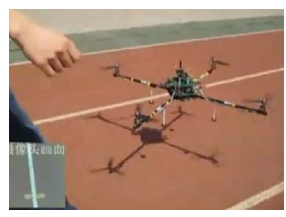

(c)

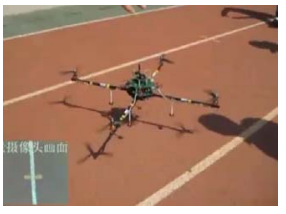

(d)

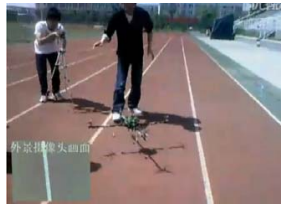

(b)

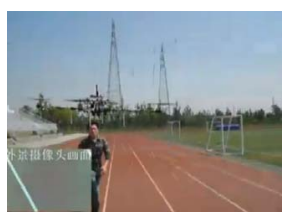

(e)

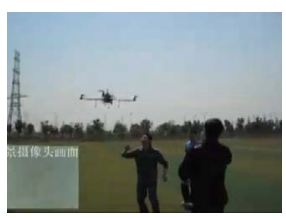

(f)

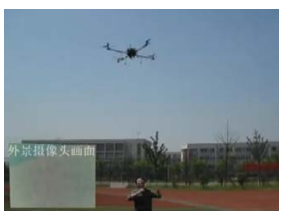

(g)

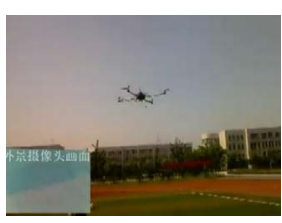

(h)

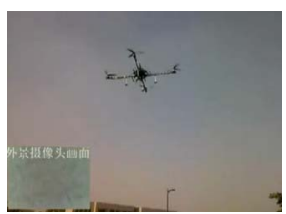

(i)

Figure 6. Outdoor experiment results

\section{CONCLUSIONS}

It is hard to go to scenes where serious natural disasters befell, biological pollution happens, and various dangers exist. So how to design a rescue vehicle which can get remote mifi control and feedback messages on time becomes a hot topic. Based on the idea above, a quadrotor aircraft, which can be used after disaster, is designed in this paper. The aircraft can implement the system remote mifi control by Bluetooth, and then it sends feedback to the monitoring center, which making the rescue after disaster really come true. The feasibility of the system is proved through the indoor and outdoor experiments.

\section{ACKNOWLEDGMENT}

The support of the project of Innovation training program for College Students of Jiangsu province ([2010] No. 16 【609】) is gratefully acknowledged.

\section{REFERENCES}

[1] Liu xiaojie. Study and Implementation of Position and Attitude Estimation for Four-rotor Mini Rotorcraft Based on Vision[D].Jilin University,2009.

[2] PORNSIN-SISIRAK T.N., LEE S.W., NASSEF H., GRASMEYER J., etc. MEMS Wing Technology for A Battery-Powered Ornithopter. Micro Electro Mechanical Systems, 2000, MEMS 2000, IEEE [C]. 2000, 1: 799-804.

[3] Liu huanyi.Study and Design of Flight Control Systems for Small Scale Quadrotors[D]:Shanghai Jiaotong University, 2009.

[4] HOU Xiang-feng,LIU Rong*, ZHOU Zhao-feng. Application of Accelerometer MMA7260 in the Gait Feature Extraction [J]. Chinese Journal of Sensors and Actuators, 2007,20(3):507-511.

[5] Kis L, Regula G, Lantos B. Design and hardware-in-the-loop test of the embedded control system of an indoor quadrotor helicopter[C]// Proceedings of 2008 International Workshop on Intelligent Solutions in Embedded Systems, 2008:1-10.

[6] LIU Zhi-jun, LV Qiang, WANG Dong -lai. Modeling And Simulation Control Of a Small-sized Quadrotor Helicopter[J]. Computer Simulation, 2010,27(7):18-20.

[7] Bouabdallah S, Noth A, Siegwart R. PID vs LQ control techniques applied to an indoor micro quadrotor[C]// Proceedings of 2004 IEEE/RSJ International Conference on Intelligent Robots and Systems (IROS 2004),2004:2451-2456. 